\title{
Are dynamic features required in signs? ${ }^{1}$
}

\author{
Rachel Channon and Harry van der Hulst
}

\section{Abstract}

This chapter argues that dynamic features are required in signs, even though they are not used in spoken languages. Hayes (1993) and others (e.g. van der Hulst 1993) have argued that they should also be eliminated from signs. But at least one dynamic class node, pathshape, is here shown to be required. This opens up the possibility that other dynamic class nodes and features are available as well, since no absolute ban on such features can be maintained. We understand dynamic features to be basic gestalt-like shape concepts that have no internal structure.

\section{Dynamic features}

The literature on the form of signs contains proposals for dynamic features ${ }^{2}$ such as 'downward' or 'upward' for directions, 'zigzag' or 'curved' for pathshapes, 'rotate' or 'pivot' for orientation changes, and 'wiggling' or 'hooking' to describe movements of the fingers. Most models use at least one or two dynamic class nodes or features such as [pathshape] or [repeat].

But are these kinds of features really needed? Since the advent of autosegmental phonology, dynamic features for spoken languages such as [delayed release] or [prenasal] have been considered superfluous because such features could be replaced by a sequence of two values for one static feature ([-continuant $] \rightarrow$ [+ continuant $]$; [+nasal $] \rightarrow$ [-nasal] $)$, or by an incompatible feature combination that requires phonetic sequencing (e.g. [-sonorant, +nasal]) for prenasalized stops). On this basis Hayes (1993) proposed removing dynamic features from sign representations.

If dynamic features for pathshapes are not used in representing signs, movements must be computed as interpolations between static points. Even the most complex movement can be understood in this way if there is no limit on the number of points that can be specified. For example, a videoclip of a person signing is actually a set of static snapshots (which the mind interprets or integrates into a moving representation). One might argue that dynamic 
characteristics are not fundamental properties of a geometric representation of a path, but rather derivative, gestalt or integrative notions. From this perspective therefore, the static 'digital' representation of movement as a set of points along a line may seem simpler, more accurate, and more fundamental.

Nevertheless, this chapter argues that dynamic features are a necessary part of the phonological representation of a sign. The primary focus here is on pathshape and secondarily on repetition features. Other arguments for specific dynamic features have been made in Channon 2002b and Mak and Tang (this volume) for repetition, Channon Crain 1996, Channon 2002a (arguments for direction features and the use of dynamic features in general), and Corina 1993 for a dynamic handshape change feature.

The chapter is organized into two major sections. The first section explains dynamic features and gives two detailed examples for a dynamic single segment model. The second section is a comparison of the two logically possible models: a dynamic model which allows dynamic features, and the non-dynamic model (anything else). The goal is to show that using a dynamic model produces simple phonetically based representations while a non-dynamic model results in logical absurdities, arbitrary phonological constraints, and excesses (such as huge numbers of segments and features for a given sign) that do not occur in the dynamic model. Note that occasionally arguments are simplified by making reasonable but unproven assumptions about sign languages. The simplifications are allowable because they are only used to make the model argued against (the non-dynamic model) more likely. All sign language examples that follow are from ASL, but the characteristics discussed are fundamental properties of representations which surely apply to all natural sign languages. For example, it is unlikely that there are sign languages without circular pathshapes, and it is unlikely that one sign language would use interpolation and arbitrary locations (as discussed below) while other languages used pathshapes.

Frequency values from the SignTyp database are used throughout the chapter. This database, which was created under NSF grant 0544944, consists of annotations for 9 datasets of 6 languages (two languages have multiple datasets of signs collected at different points in time) with a total of 11,956 signs as shown in Table 1 . These were originally separate datasets from different coders using different annotation systems, which were converted to the SignTyp coding system and structure and added to the database. Some datasets are more complete than others, in part because some contained more information and in part because conversion is not yet complete for all datasets. More information and a download are available at http://www.ldc. upenn.edu/signtyp/downloads/. 
Table 1. SignTyp database

\begin{tabular}{lllr}
\hline SIL Codes & Sign language & Dataset (original coder or source) & Signs \\
\hline ase & American & Hara/Stokoe & 1890 \\
ase & Old American & Long & 1549 \\
ase & American & Rozelle & 656 \\
dse & Netherlands & SignPhon & 3080 \\
fse & Old Finnish & Rozelle & 609 \\
fse & Finnish & Rozelle & 354 \\
jsl & Japanese & Hara & 2516 \\
kvk & Korean & Rozelle & 614 \\
nzs & New Zealand & Rozelle & 688 \\
\hline Total & & & 11956 \\
\hline
\end{tabular}

\section{What are dynamic features?}

Dynamic features are characteristics of motion such as direction, pathshape, repetition, size, speed, tension, or change in shape or orientation. These features occur over a discernible period of time. While motion cannot be directly seen in a snapshot, there is a way in which at least pathshape and repetition are potentially atemporally visible. Imagine standing on top of a building and looking down at a landscape with a circular path that you plan to walk on. You can see your future path ${ }^{3}$ as an atemporal whole, without regard to direction or where you plan to begin or end on the path. Similarly, visual perception of repetition can be atemporal as in looking at a garden or work of art and easily picking out various types of repeated elements. These repeated elements can be perceived without any ordering or temporal reference (such as to when the items were placed in position). Contrast this with possible auditory dynamic features: although one may speak of a voice as falling or rising and can perceive that some sound has been repeated, these perceptions have a metaphorical quality to them (in that a term like 'rising' refers to an upward movement, and so on) and/or are clearly accompanied by an understanding that time is involved. This difference in visual and audible perception may be reflected in the proposed difference in the use of dynamic features in sign and speech ${ }^{4}$. 
A significant characteristic of many dynamic features is their ability to sequence static (i.e. non-dynamic) features. Referring back to the analogy of a person observing a path from above, if you know that you will walk around the path clockwise and know either your beginning or ending point, then for any two points along the path, you can determine their sequence - which point you will pass through first or second. Similar sequencing information is available through phonological features such as down, opening, or pronating. If there is a location on the chin and the chest, and a direction down, then the two locations must be sequenced chin $\rightarrow$ chest. If there are two handshapes such as an open spread hand and a fist and a handshape change feature opening, then the two handshapes must be sequenced fist $\rightarrow$ open spread hand. If there are two orientations of the palm such as palm facing floor and palm facing up (with elbow flexed) and an orientation change feature pronating then the two orientations must be sequenced palm facing $u p \rightarrow$ palm facing floor. While there are a few exceptions, dynamic features usually cannot sequence more than two features, but as has been noted by a number of phonologists, signs rarely have more than two distinct values of any particular feature type: for example not more than two distinct locations (Wilbur 1993).

Although dynamic features can sequence static features, it appears to be even more common that they allow for the omission of static features. For example, if there is a location on the chest and a direction out, then it is clear that the hand begins at the chest and moves out, but no final location needs to be specified. There simply is a location where the movement takes place. Likewise, if there is a dynamic orientation feature pronating, then the hand must begin in a neutral or supine position and move to a pronated position, and neither the initial nor final orientation of the arm needs to be specified.

When static features are omitted, the resulting locations, orientations, handshapes and other characteristics will be somewhat vague or allow for several similar choices: if location is chest and direction is out, then the final location of the hand is somewhere out from the chest, but it could be a very short or very long distance out without changing the meaning of the sign. In the second example in the previous paragraph, the initial and/or final orientations may vary considerably, providing that it allows for a pronating action of the arm. As discussed in more detail below, this is in fact what is often seen in signs - that while some static features are relatively fixed, others may vary considerably, because these characteristics are not a part of the phonological representation but are the result of dynamic phonological features. One important characteristic of the dynamic model is its ability to explain this difference between more fixed and more flexible characteristics. More 
examples are given below in the sample signs.

As a consequence of the nature of dynamic features and especially this sequencing ability, representations using dynamic features are normally extremely simple and all non-compound signs can be represented within a single segment.

\subsection{Examples of single segment dynamic representations}

Although the primary point of this chapter is to show the representational problems that occur when dynamic features are not allowed, it may be helpful to show some of the positive results of using them. This section shows that the dynamic model allows compact non-redundant representations because many characteristics ${ }^{5}$ can be determined by implication from other features, or by assuming the unmarked or phonetically determined value of a feature. The model used here assumes a single segment representation with dynamic features as in Channon 2002a. Two examples with accompanying explanations are given: KNOW and PLEASE.

In what follows, omitted values are sometimes referred to as implied and sometimes as default, although it is not clear that there is any real distinction between these two terms. Implied is used when there is a clear physical explanation for why a particular characteristic is omitted, and this explanation depends on the value of a feature(s) in the representation. Default is used when the value is omitted primarily because it is by far the most common value in SignTyp, either overall, or for a pertinent subset (such as most common orientation for signs overall or most common orientation for signs made in a particular location). Most or all defaults probably have a physical explanation although not necessarily an implicational explanation. Note that both default and implied values can be overridden by specifically adding some marked value to the representation.

\subsubsection{Representation example: KNOW}

Figure 1 provides the illustration for KNOW and Figure 2 the full dynamic representation. Even this representation is simpler than what would be seen in most multisegmental models, but it is still redundant.

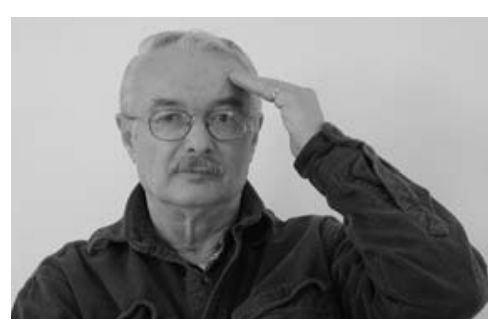

Figure 1. KNOW 


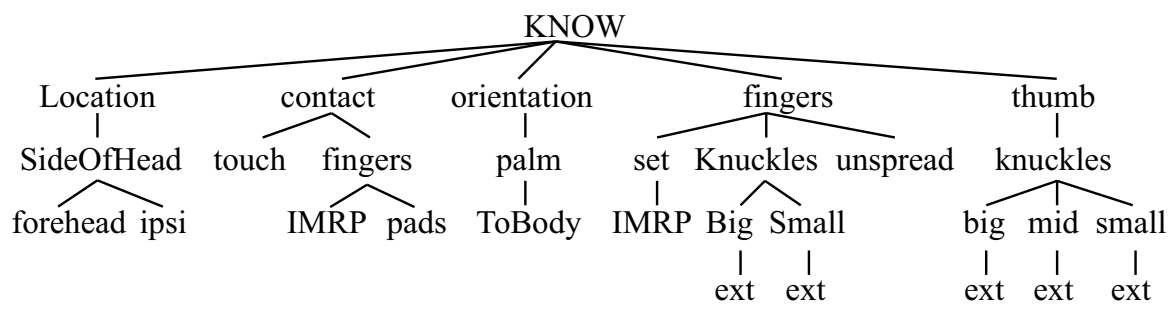

Figure 2. KNOW - Redundant dynamic model representation

A word description of Figure 2 is: KNOW is made at the ipsilateral side of the forehead. The hand touches the location with the pads of all fingers. The palm faces the body. The handshape has all fingers extended and unspread, with the thumb extended. Omitted information includes: repetition (none), pathshape (none), second hand (none) and nonmanual expression (none).

Figure 3 shows a non-redundant representation with simplified location information, contacting part information retained, and no specifications for handshape, movement or orientation. The claim is that deleted information falls out from various default values and implications. The following explanations discuss all characteristics in turn, and give the reasoning behind what information is kept or removed. (The focus of this chapter is primarily on redundancy and dynamic features, so few supporting arguments are provided for the chosen dependencies, which are tentative and may need revision.)

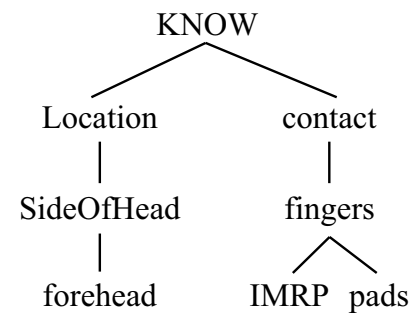

Figure 3. KNOW - Nonredundant Dynamic model representation

\subsubsection{Location}

Forehead is shown as a dependent of SideOfHead because in many cases, KNOW is actually performed lower on the head but always on the side. This effect can be explained as a deletion of forehead, leaving SideOfHead.

Ipsilateral is considered redundant because it is (by definition) the closest location to the acting hand, and therefore on the face, this is the easiest side for the hand to contact. However, the data from SignTyp is slightly ambiguous on this issue. SignTyp currently has two datasets that specify locations 
in sufficient detail to be of use in this question: the SignPhon NGT and the Long ASL datasets. If all signs with information about locations are included, as in Table 2, then the preference for ipsilateral locations as opposed to central or contralateral is clear. If only signs made on the body are included, as in Table 3, or only signs made on the head, as in Table 4, the preference for ipsilateral is still clear. But if only signs made on the forehead are considered, as in Table 5, the situation is a little murkier. The SignPhon data maintains the ipsilateral preference, but the Long data shows a preference for a central location as opposed to an ipsilateral one. More data is certainly needed to resolve this variability, but it seems likely that the Long data represents a younger, as well as a more formal, language set, where there is an opposing tendency to symmetry and centrality which over time, gives way to the physically easier and visually clearer peripheral locations. A picture and description of the sign KNOW from the Long data (Figure 4) shows that in this early 1900s incarnation of ASL, the sign was made centrally (and with a much flatter handshape than is the norm now).

Table 2. Laterality preferences for all locations

\begin{tabular}{lrrr}
\hline laterality & Long & SignPhon & Total \\
\hline ipsilateral & $61.7 \%$ & $78.5 \%$ & $77.7 \%$ \\
center & $23.0 \%$ & $15.9 \%$ & $16.2 \%$ \\
contralateral & $15.3 \%$ & $5.6 \%$ & $6.0 \%$ \\
$\boldsymbol{n}$ & 222 & 4566 & 4788 \\
\hline
\end{tabular}

Table 3. Laterality preferences for all body locations

\begin{tabular}{lrrr}
\hline laterality & Long & SignPhon & Total \\
\hline ipsilateral & $60.5 \%$ & $61.1 \%$ & $61.1 \%$ \\
center & $26.5 \%$ & $29.1 \%$ & $28.7 \%$ \\
contralateral & $13.0 \%$ & $9.8 \%$ & $10.2 \%$ \\
$\boldsymbol{n}$ & 185 & 1153 & 1338 \\
\hline
\end{tabular}

Table 4. Laterality preferences for all head locations

\begin{tabular}{lrrr}
\hline laterality & Long & SignPhon & Total \\
\hline ipsilateral & $63.7 \%$ & $96.6 \%$ & $90.1 \%$ \\
center & $35.5 \%$ & $0.0 \%$ & $7.0 \%$ \\
contralateral & $0.8 \%$ & $3.4 \%$ & $2.9 \%$ \\
$\boldsymbol{n}$ & 124 & 502 & 626 \\
\hline
\end{tabular}


Table 5. Laterality preferences for all forehead locations

\begin{tabular}{lrrr}
\hline laterality & Long & SignPhon & Total \\
\hline ipsilateral & $36.4 \%$ & $96.3 \%$ & $87.1 \%$ \\
center & $63.6 \%$ & $0.0 \%$ & $9.8 \%$ \\
contralateral & $0.0 \%$ & $3.7 \%$ & $3.1 \%$ \\
$\boldsymbol{n}$ & 44 & 243 & 287 \\
\hline
\end{tabular}

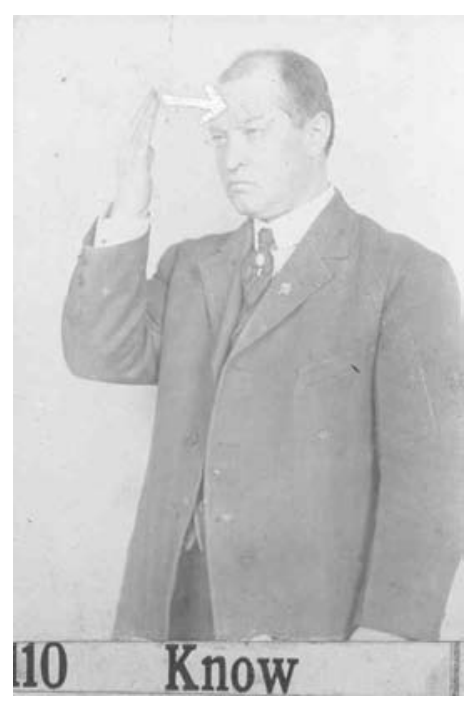

Figure 4. KNOW in Long dataset

Thus, although overall, there is a preference for ipsilateral locations, more data is needed. For now, ipsilateral is considered the default location, while leaving open the possibility that it is a true phonological feature and must be included in the representation.

\subsubsection{Contact part and type}

Contacting part is included. Contact type is omitted based on two reasonable assumptions. First, if there is a contacting part, then there must be contact with the specified location(s). Second, the unmarked contact type is assumed to be with the hand moving toward the location(s) and lightly touching or tapping it. 


\subsubsection{Orientation}

All orientation information is omitted because it is implied by the contacting part (fingerpads). Two basic orientations are observed: the palm faces the body and the fingers point either up or toward the body. These two orientations are the physically easiest orientations for this sign. Other orientations of the hand would be impossible with fingerpad contact or would require unnecessary expenditures of energy or move the arm into uncomfortable positions:

- Palm facing out toward the viewer: Impossible if the fingerpads must contact the forehead.

- Fingers pointing contralaterally: Requires an awkward twist of the wrist and/or raising the entire arm out of neutral position which is less energy-efficient.

- Fingers pointing down: Less energy-efficient since it would require raising the elbow to about head height.

Note that the absence of a feature for a particular representation does not imply that it is always absent. So while impossible orientations are of course ruled out (for signs with the same handshape and location), orientations that are less energy efficient are not ruled out for other signs. But if such an orientation does occur, it must either be specified in the sign or be predicted by some other combination of characteristics. For example, the marked orientation of ASL LION (hand with spread, extended fingers pointing down traces a "mane" from forehead toward back of head) must be a part of the representation. As another example, suppose that a sign is made with the extended thumb contacting the forehead. In this case, the unmarked orientation would be for the palm to face contralaterally (as in FATHER and other signs).

The reader can easily verify these unmarked orientations even without any knowledge of sign languages. Simply touch the forehead with the pads of all fingers or with the tip of the thumb and observe which orientations are possible and which ones are easy.

\subsubsection{Fingerset and posture}

The fingerset and posture are redundant, because the contacting part implies fingerset, posture (and orientation). Note that the reverse is not true: fingerset, posture and orientation do not imply contacting part: the given fingerset and 
orientation could have a contact point on the heel of the hand, the entire surface of the palmside of the hand, the palm of the hand or the entire surface of the fingers.

Given that fingertips is the contacting part, the fingerset must be IMRP (index middle ring and pinky) acting as a unit. Finger posture is slightly more variable. Possible postures are: extended, bent, hooked, curved and closed ${ }^{6}$. Closed and hooked handshapes are eliminated because they do not allow the fingerpads to contact the forehead. Curved, bent and extended handshapes all allow contact with all the finger pads, and all these postures are seen. Note that omitting finger posture from the representation is therefore not only possible, but more accurate, since it allows for the actual posture variation.

Although contacting part does not always predicts the fingerset and posture, it is likely that for signs that contact the body, the contacting part may be included in the representation far more often than other handshape information. Contacting part is more constraining and predictive than the notion of selected fingers. Selected fingers lightly constrain but cannot predict the contacting part for most signs. For example, even a relatively restricted handshape such as the F hand (index and thumb contact, middle ring and pinky extended) has more than one possible contacting part in ASL: in the sign CAT, the fingertips contact, in the sign IMPORTANT the sides of the fingers contact and in the sign COUNT the side of the thumb contacts. And of course, more common handshapes, like the flat hand, have many possible contacting parts.

Contacting part may sufficiently limit the choices of fingerset and posture that in conjunction with unmarked choices for thumb posture, digit crossing and spreading (see below), no further information about the hand is needed - as in KNOW. But there are certainly signs with contact where the limitation is insufficient ${ }^{7}$, and further phonological features must be included. And signs without contact, and therefore no contacting part, would usually need to include handshape features.

\subsubsection{Finger spreading}

Unspread fingers is assumed as the default spreading value, based on Table 6 which shows that unspread is strongly preferred for handshapes with all fingers acting together in an extended, bent or curved posture. 
Table 6. SignTyp spreading values for strong IMRP handshapes with extended, bent or curved postures

\begin{tabular}{lll}
\hline & \multicolumn{2}{l}{ Strong handshapes } \\
\hline & $\boldsymbol{n}$ & $\%$ \\
\hline unspread & 3944 & $73.3 \%$ \\
spread & 1435 & $26.7 \%$ \\
Total & 5379 & $100.0 \%$ \\
\hline
\end{tabular}

\subsubsection{Thumb}

The thumb has several elements:

Posture: which if any knuckles are flexed.

Contact point: where the thumb contacts the fingers (tips, pads, palm side of fingers, etc.)

Crossing: whether the thumb is underneath the fingers (when the fingers are not extended)

Opposition: the large knuckles of the thumb move it into opposition with the plane of the fingers them

The default values for extended, bent or curved handshapes can be assumed to be uncrossed with no contact point. A hooked posture of the thumb is difficult and is rarely seen except when the fingers are hooked. An opposed thumb posture is a bad choice for any sign on the forehead since one runs a risk of poking the thumb into the eye. A posture with the thumb folded into the palm is physically possible, but there are several objections. First although the achieved posture is not dangerous to the eye, moving into such a posture as the hand moves toward the forehead could be risky. Secondly, the posture appears to be physically slightly more difficult than either an extended thumb or a thumb next to the index. Thirdly, this thumb posture is uncommon - it constitutes only $3 \%$ of thumb postures for strong hands with unspread IMRP handshapes with extended, bent, or curved postures. When a posture is uncommon, it suggests that for it to appear, it needs to be phonologically specified.

This leaves two choices: the extended thumb or the thumb next to the index. While both appear to be acceptable for KNOW, observation suggests that the extended thumb is more common. Table 7 shows the preference patterns by spreading choice for strong handshapes in signs with all fingers selected (IMRP), with bent, curved or extended finger postures. Although the 
numbers are small, unspread IMRP signs with bent or curved postures prefer the thumb closed next to the index, but for all other combinations of posture and spreading, the preference is for an extended thumb.

The apparent unspread nature of KNOW may actually be better understood as an indeterminate element - that the absence of a spread value in Figure 3 does not equate to a default value of unspread, but rather the hand is neither spread nor unspread. Phonetically, this appears to be an unspread handshape, but it is actually the resting position of the hand when no choice has been made - the fingers slightly but inconspicuously separated, neither widely spread nor tensely contacting each other. In this default position, the thumb will be slightly extended in the same plane as the palm. In other words, the most accurate way of understanding both spreading and thumb posture in KNOW is to say that they are unspecified (as in the representation) and the resultant handshape has the phonetically most relaxed choices for both spreading and thumb posture, which happen to closely resemble the phonological choices of unspread handshape and extended thumb. For now however, it seems sufficient to show that in this sign, thumb posture is not fully determined, and can vary between at least two positions - next to the index or extended in the same plane as the palm.

Table 7. Thumb posture data for strong hand in SignTyp

\begin{tabular}{llrcc}
\hline $\begin{array}{l}\text { Finger } \\
\text { Posture }\end{array}$ & Finger & $n$ & $\begin{array}{c}\text { \% thumb extended in } \\
\text { same plane as palm }\end{array}$ & $\begin{array}{c}\text { \% thumb closed } \\
\text { next to index }\end{array}$ \\
\hline Bent & Unspread & 346 & 33.8 & 66.2 \\
& Spread & 44 & 50.0 & 50.0 \\
Curved & Unspread & 183 & 10.9 & 89.1 \\
& Spread & 137 & 99.3 & 0.7 \\
\multirow{2}{*}{ Extended } & Unspread & 2184 & 90.8 & 9.2 \\
& Spread & 976 & 95.6 & 4.4 \\
Total & Unspread & 2713 & 83.6 & 16.4 \\
Total & Spread & 1157 & 94.3 & 5.7 \\
\hline Total & Spread $\&$ & $\mathbf{3 8 7 0}$ & $\mathbf{8 6 . 0}$ & $\mathbf{1 4 . 0}$ \\
& Unspread & & & \\
\hline
\end{tabular}




\subsubsection{Representation example: PLEASE}

The second example is PLEASE (or ENJOY), which will be further referenced in the second part of this chapter. The sign is shown in Figure 5 and the proposed dynamic representation in Figure 6. PLEASE moves the palm side of the hand on the chest in a repeating circling pathshape.

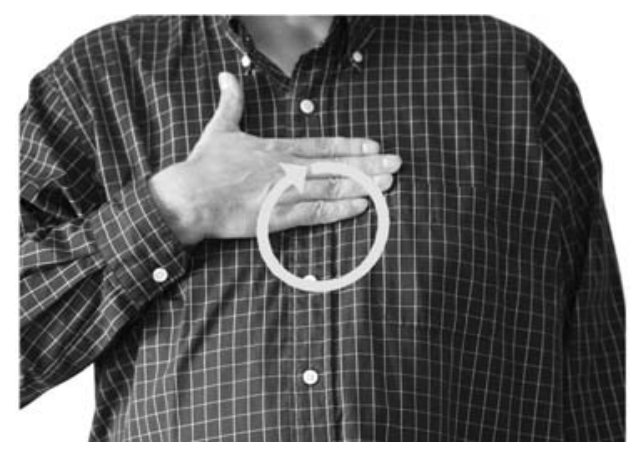

Figure 5. PLEASE in a dynamic model

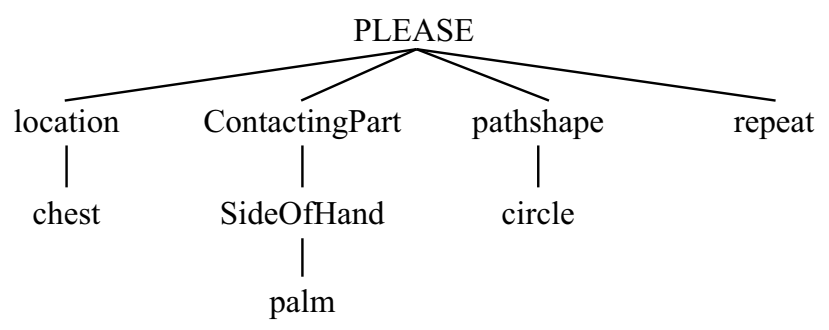

Figure 6. PLEASE - dynamic model representation

Location. It is not necessary to specify the location in any more detail than chest. The hand moves through various points of the chest, but these are all predictable from a circular pathshape ${ }^{8}$.

Handshape and orientation. Fingerset, posture, thumb values, and orientation can be deduced from the contacting part. If the strong hand contacts the chest with the palm side of the hand, the orientation will have the palm facing the body as required to produce this contact. For the same reason, the hand must be flat. The lack of spreading is the default value (as in KNOW). The thumb must be either next to the index or extended in the same plane, with essentially the same reasoning as in KNOW.

Contact type. It is possible that an additional feature is required: type of contact which would be specified as rubbing or continuous. It is omitted here because this can probably be deduced from the pathshape and location. If the 
hand circles on the chest and there is contact, the natural contact type would be continuous. Nevertheless, if it were shown to be necessary, a contact type could be added to PLEASE. This would be the case if a sign were found that contacted the chest in a circular path but had a vertical or horizontal path. Such a sign would be similar to HAPPY ${ }^{9}$ which moves in a vertical plane contacting the chest briefly as it moves up repeatedly. However, HAPPY does not have a circular path, so it is not the minimal pair needed to show that contact type is required for PLEASE. So unless this type of sign is identified, contact type is not needed here.

Direction. A direction feature is omitted on the grounds that there are no minimal pairs in ASL caused by a change in the direction of a circle, and that the default circling action in the lateral plane is an up-contralateral-downipsilateral motion.

\subsection{Example summary}

To sum up, representations for signs in a dynamic model vary in two significant ways: the number and kind of features. The simplest signs such as KNOW and PLEASE have a minimal representation of a few features. Others may have more numerous features, but even these complex signs have many omitted characteristics.

The specific features required or omitted vary from sign to sign. Brentari \& Eccarius (this volume) propose something similar when they observe that in the core lexicon, signers do not distinguish between flat and circular postures of the thumb and fingers in $\mathrm{O}$ and $\mathrm{F}$ handshapes, although they do distinguish between these versions for flat or rounded classifiers, or (for $\mathrm{O}$ handshapes only) when it is an initialized sign. In other words, something that is a phonetic variant in one situation becomes a phonological distinction in another.

Most parameters have little detail. Very few signs (probably none) have a fully specified handshape indicating exactly how the knuckles of each digit behave and the relationship of thumb and finger. Different dynamic features appear in different signs, and most signs probably have no or only one dynamic features. The assumption is that dynamic features (like static features) have a cost, and probably are more costly than static features, but no research has been done yet on this issue.

The dynamic model per se does not require that features/characteristics be left out of the representations for a particular sign. Any sign could be represented with full details about location, handshape, orientation, movement, 
contact and non-manuals similar to KNOW in Figure 2. But with dynamic features, it becomes possible and reasonable to simplify the representations as shown in Figure 3.

The point is worth stressing, because the proposed simplified representations shown above may turn out to be incorrect in some or even many details. But even if the current choices are incorrect, this still would not invalidate the argument for a dynamic model. Features for a particular sign or a group of signs might need adjustment, but the choice of features and the choice of model are separate (though related) inquiries.

Does the use of dynamic features necessarily imply a single segment model? If the model does not use dynamic features, a multiple segment representation (or at least a representation with timing units of some kind) is absolutely required in order to temporally order features. But if the model uses dynamic features, the answer becomes more complex: a single segment model becomes possible but ruling out a multisegment model is a matter of multiple arguments. Each argument alone might not be sufficient to show that a single segment model is required, but the cumulative weight of various arguments may be more persuasive. In many signs, no significant problems can be shown regardless of whether a multisegment or single segment representation with dynamic features is used. However, Channon 2002a has shown that for significant sets of signs, a multiple segment model produces peculiarities, excessive feature cost, overgeneration of representations for the same sign, overgeneration of unattested forms, and logical absurdities. Two arguments are summarized here:

1. Signs with reversing movement where the reversal is meaningful require only a change of feature to produce the change in meaning in the single segment model. An example is STATUS-CHANGE (or IMPROVE/GET-WORSE) which contacts the arm repeatedly while moving up or down to indicate improvement or deterioration. But in a multisegment model, the change in meaning requires a reversal of segments, such as $\mathrm{S} 1 \rightarrow \mathrm{S} 2 \rightarrow \mathrm{S} 3$ switching to $\mathrm{S} 3 \rightarrow \mathrm{S} 2 \rightarrow \mathrm{S} 1$, a method of producing meaning change never seen in spoken languages ( $d o g$ is not the opposite of God).

2. In other signs, such as DEAF which can be signed by contacting the ear and then the mouth OR the mouth and then the ear, a multiple segment model must use two distinct representations $(\mathrm{S} 1 \rightarrow \mathrm{S} 2$ and $\mathrm{S} 2 \rightarrow$ $\mathrm{S} 1)$ for the same meaning, and therefore overgenerates representations. The single segment model uses only one representation which has no direction feature. Because there is no segmental sequence and 
no sequence provided by a direction feature, the particular direction that occurs in the utterance will be determined by phonetic or sociolinguistic factors, by contiguity (previous or succeeding signs), personal preference, or even chance.

See Channon 2002a and 2002b for fuller explanations of these arguments as well as many other arguments for a single segment model.

Even when a single segment model is shown to be required, the exact nature of the model is still an open question. Van der Hulst (1993) and Channon (2002a) provide somewhat different versions of a single segment model. The Channon model is used here, but this choice is not justified beyond what has already been said. Many issues about the correct phonological model for signs remain to be resolved. Here, the primary goals are to show that dynamic features both simplify and are required in the representation of many signs.

\section{Required dynamic features: Pathshape and repetition}

Two dynamic features or feature groups are commonly used by sign language phonologists: pathshape and repetition. For example, Brentari (1998:50) uses several such features which she calls movement shape: [circle], [straight], [arc], [tracing] as well as repeat and alternating features. Sandler (1993) has [arc] and [redup] features, the model of van der Hulst (1993), Crasborn (2001) and van der Kooij (2002), uses "manner features" similarly, Hansen (this volume) uses [oscillating] and various circling features, Mak \& Tang (this volume) have two repetition features [repeat] and [return], and Channon (2002a and 2002b) uses [repeat], several values of [pathshape] and other dynamic features. Although Liddell and Johnson (1989) do not use a repetition feature, they do have some pathshapes.

Pathshape (and concomitant repetition issues) has been selected as the "poster child" for dynamic features because of this use. Other dynamic features, such as direction (Channon Crain 1996, Channon 2002a) or handshape opening/closing (Corina 1993), are not as widely accepted.

In spite of the common use of dynamic repetition and pathshape features, there has not been much discussion of why dynamic features as a class are needed, what problems occur if they are not used, and what the implications for segmental structure are if they are used. One discussion is in Padden $\&$ Perlmutter (1987). Although they adopted a segmental approach, they have an interesting appendix pointing out the problems with alternating and 
trilling for such an approach. They observe that trilled movement, which has an indefinite number of repetitions, must be represented as a dynamic feature [trill] instead of a series of segments because otherwise it "would lead to the absurd result that the phonological representation consists of a different number of segments in different performances of the sign". They note also that a multisegmental representation is problematic with dynamic features because they cannot be associated with a single segment. They show that alternating in the Liddell \& Johnson (1984) model is not a feature, but a characteristic of the temporal arrangement of the segmental tiers of the two hands, and that therefore if alternating signs change to trilled signs, this change cannot be easily represented as a change from [alternating] to [trill]. Instead it requires wholesale changes to the structure of the sign, and peculiar complex rules that specify that signs with a particular temporal arrangement of the segments of each hand tier change to a sign with a single $\mathrm{H}$ segment and a feature [trill].

The problem of multiple representations with a "different number of segments in different performances" is discussed at greater length in Channon $2002 \mathrm{a}$ and 2002b. Channon 2002b argues that a repetition feature is required primarily because number of repetitions is not contrastive in signs (except for some classifier predicates where it is iconic). Repetition most commonly occurs just once, but two or more repetitions are also well formed utterances. A model with a repetition feature can use one representation for all of these forms. But a model without one overgenerates representations: every utterance with a different numbers of repetitions must have separate representations, even though such utterances are all considered to be the same sign. In 2002a she shows that many other sets of signs also overgenerate representations, in a multiple segment model and this problem is best resolved by using direction features and a single segment model.

Generating non-contrastive but separate representations for the same sign is one type of overgeneration. The second type is overgeneration of forms that do not exist in the language. An example of the second type is that any multisegmental model (dynamic or non-dynamic) for sign languages predicts that forms should exist in the language with indeterminately long location sequences, or more generally, indeterminately long segment strings. Examples of such strings are nose-ear-forehead-mouth, or ear-nose-forehead-chestmouth-ear. But sign languages exhibit a systematic gap - these long location strings do not exist in simple signs, even though such sequences would be perfectly acceptable in strings of signs. Even compounds show only very restricted location strings. Therefore multisegmental models of signs overgenerate non-existing forms (see Channon 2002a for further discussion). 
4.1 Representing pathshape with multiple locations along the path: interpolated locations

If all paths were single straight lines, then a pathshape feature would not be needed. But in fact there are many other possible paths including crossshaped, rectangular, 7, arc, semi-circle, circle and spiral path.

A non-dynamic model can only represent a pathshape by listing points along the path. Of course, for any line or line segment, there are an infinite number of possible points, making it impossible to actually specify every point along the path. So any nondynamic model must use interpolation to describe pathshapes. Straight lines, 7 paths, arcs, semi-circles and circles are considered below in turn. Other possible paths are omitted from consideration to make the argument simpler - the logic of the argument is not affected.

Straight lines. The number of points and the type of interpolation (straight or curved) for all possible pathshapes must be established. For a straight line, the beginning and ending points have a natural salience that make them suitable to select as the phonological locations required by the non-dynamic model. A first approximation of an Interpolation Constraint might be:

1) If all phonological locations are collinear, then the interpolation method is linear.

7 pathshapes. The naturally salient points for a 7 pathshape (two straight line paths joined at a somewhat acute angle) would be the two end points and the point at the angle. The Interpolation Constraint is still linear, but needs adjustment:

2) If there are either two or three distinct phonological locations, then the interpolation method is linear.

Note that a repetition of the same location does not count as distinct. For example $a a b, a b a, a b a b, a b b a$ all have only two distinct locations.

Arcs. To distinguish arcs from straight lines or 7 shapes requires a different number of distinct phonological locations. The smallest possible set of distinct locations is 4 . The question now arises: which 4 ? The beginning and ending points of an arc are naturally salient, but where should the other two points be located? In an arcing sign such as DAY, the hand moves in 
a smooth, continuous curve from start to finish. If there were four salient points, one would expect to see a movement which would look something like the following: the hand moves smoothly from the beginning point to a point along the arc, pauses, moves to a second point along the arc, pauses, and moves to the final point of the arc. Since this is not what is seen, and there is no other natural indication of salience, then the two interior locations must be arbitrarily selected.

For arcs, semi-circles and circles, a curvilinear interpolation method will be needed. The adjusted Interpolation Constraint is:

3) If there are either two or three distinct phonological locations, then the interpolation method is linear. If there are four distinct phonological locations, then the interpolation method is curvilinear and the path is an arc.

Semi-circles. Again, a distinct number of phonological locations is required, and since 2, 3 and 4 have already been used, the next possible set is 5. Again, as with arcs, there are only two naturally salient locations, so three interior locations must be arbitrarily selected. The Interpolation Constraint is now:

4) If there are either two or three distinct phonological locations, then the interpolation method is linear, otherwise it is curvilinear. If there are four distinct phonological locations, then the path is an arc. If there are five distinct phonological locations, then the path is a semicircle.

Circles. The smallest number of distinct locations available is 6 , so the circle must have 6 locations along it. Since a circle has no naturally salient points, all 6 locations must be arbitrarily selected (as shown in Figure 7). The final Interpolation Constraint will look like ${ }^{10}$ :

5) If there are either two or three distinct phonological locations $a, b$ and $c$, then the interpolation method is linear, otherwise it is curvilinear. If there are four distinct phonological locations, then the path is an arc. If there are five distinct phonological locations, then the path is a semicircle. If there are six distinct phonological locations, then the path is a circle.

Note that 5) must be a phonological constraint, because there are no possible phonetic characteristics that explain such a magical set of inferences. 


\subsection{Problems of a nondynamic model}

The remainder of this chapter focuses on the sign PLEASE as an example to show the implications of rejecting dynamic pathshape and repetition. PLEASE was shown above in Figure 5 with its accompanying dynamic model representation in Figure 6. The same sign is shown below in Figure 7 as understood in a nondynamic model, and one of its possible accompanying non-dynamic representations is shown in Figure 8. Note that an additional representation with even more segments is required for every additional repetition of the circling movement. Since this is a circling sign, constraint 5) above requires that six arbitrary phonological locations on the chest be selected to allow for a circling pathshape.

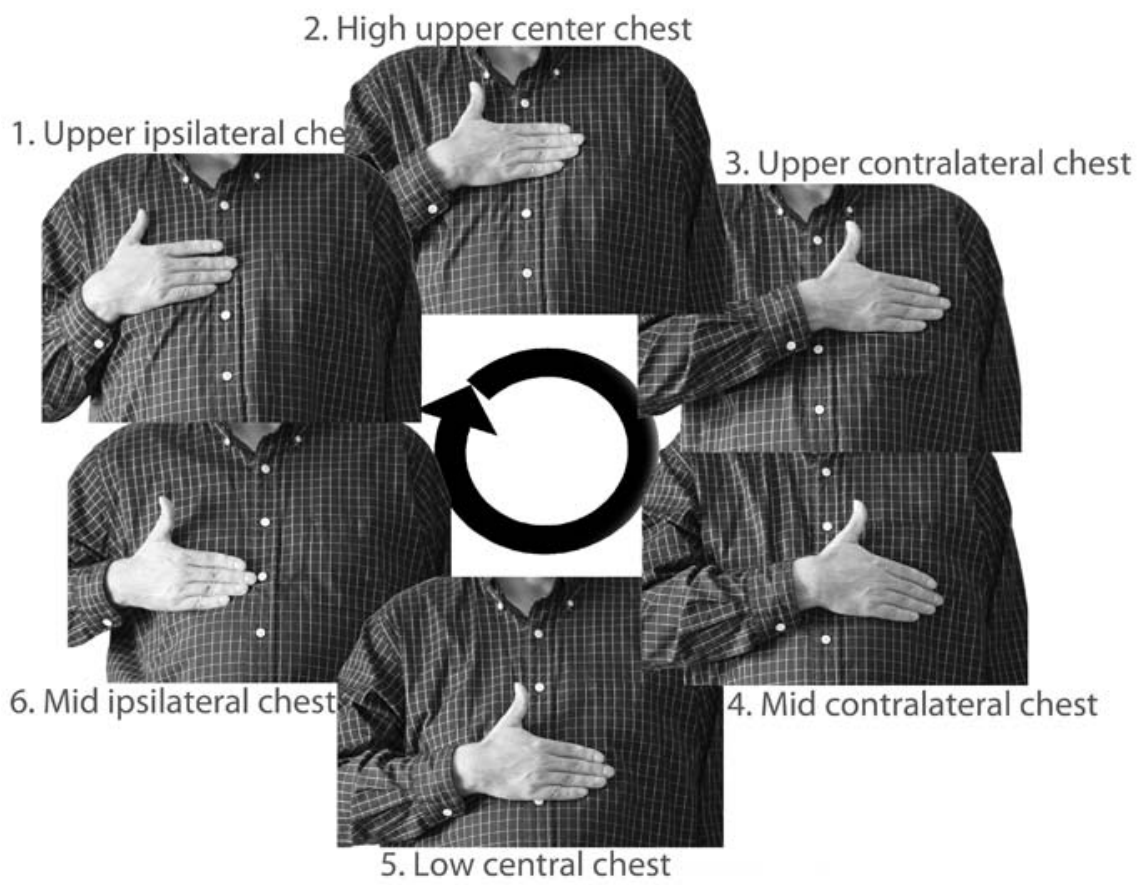

Figure 7. PLEASE in a non-dynamic model 


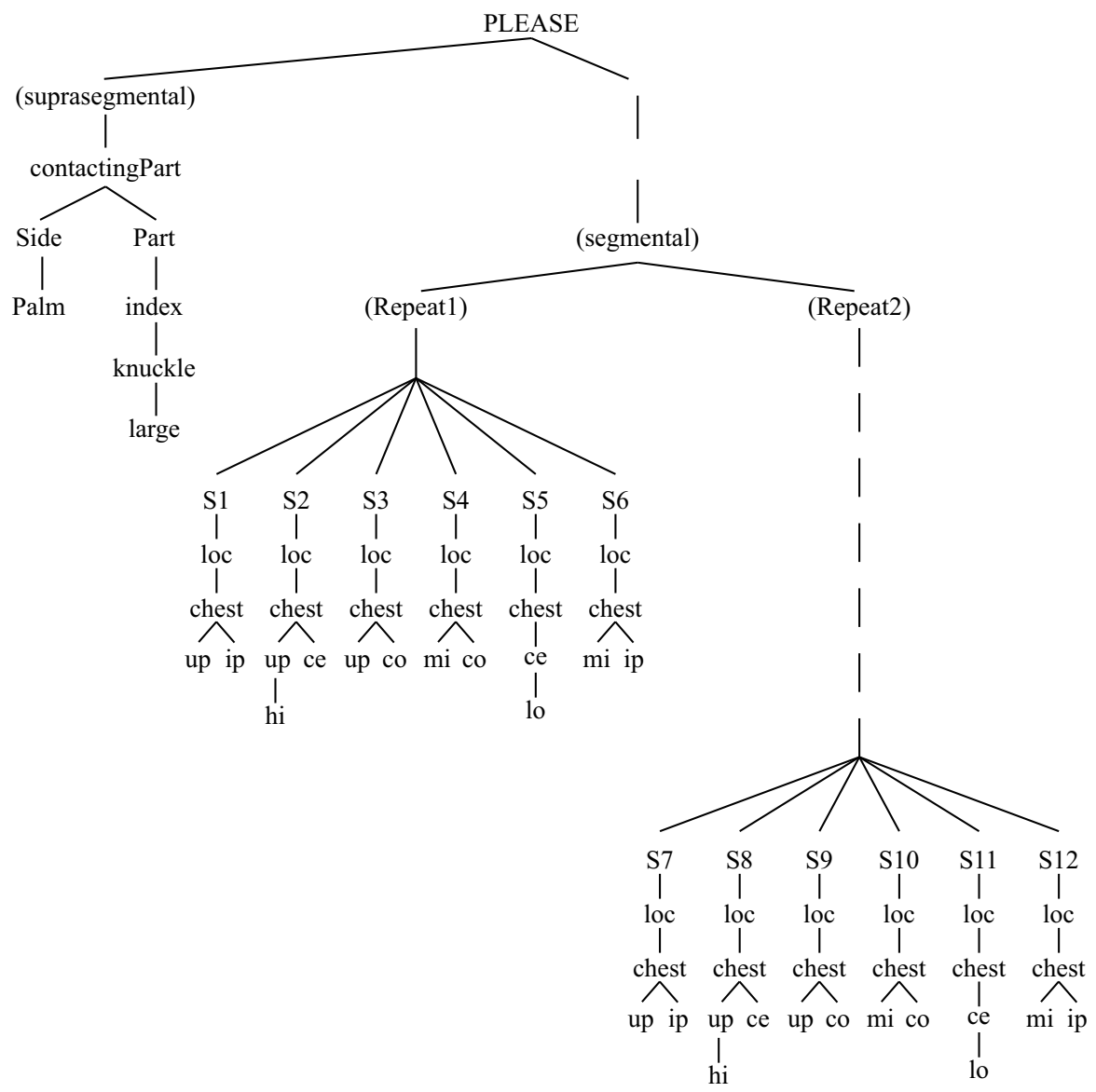

Figure 8. Nondynamic representation of an utterance of PLEASE with one repetition of path

Abbreviations: $\mathrm{S}=$ segment, up=upper, ip=ipsilateral, $\mathrm{mi}=$ middle, $c e=$ center, $c o=$ contralateral. Note: The nodes suprasegmental, segmental, Repeat1 and Repeat2 are for convenience of grouping only and are not assumed to be a real part of the representation. The placement of Repeat 2 below Repeat 1 is only because of space constraints.

As the two representations (Figure 6 and Figure 8) show, both models have one similarity: they each specify that chest contact is made with the palm side of the hand. The models differ as shown in Table 8. 
Table 8. Differences between nondynamic and dynamic models

\begin{tabular}{l|l}
\hline Nondynamic model & Dynamic model \\
\hline $\begin{array}{l}\text { Arbitrarily selected contacting part. When the palm contacts } \\
\text { and circles on the chest, different parts of the hand contact } \\
\text { different parts of the chest. For example, at the highest point of } \\
\text { the circling action, the index contacts the upper chest, but the } \\
\text { pinky contacts the mid-chest (exact contact points may vary }\end{array}$ & \\
in different performances). Therefore, in order to correctly \\
specify the location, the part of the strong hand that contacts \\
the locations is arbitrarily selected as the large knuckle of \\
the index. The selection is arbitrary because nothing in the \\
handshape marks out the index or its knuckle (in contrast to a \\
handshape such as index finger extended from a fist where the \\
index is clearly distinct from the other fingers ${ }^{11}$ ).
\end{tabular}


with all segments. Problems with this method would occur if contact features changed during the sign, as in PANCAKE ${ }^{13}$ where the contact changes from the back of the hand to the palm of the hand, or if the sign changed between contact and noncontact with the location as in a sign like HAPPY. The problem is that the suprasegmental spreading of the contact features would have to be directed to specific segments and not automatically spread to all segments. A suprasegmental level such as this would require considerable theoretical support to justify such a change. A second alternative would be to reinsert the suprasegmental elements in the segmental area, arbitrarily associated to one of the 12 segments. A third alternative would be to add these features to each segment, greatly increasing the number of features and the redundancy of the model.

Table 9 gives side-by-side word descriptions based on the two phonological models to demonstrate the differences in complexity and arbitrariness.

Table 9. Description of PLEASE in two different models

\begin{tabular}{l|l}
\hline $\begin{array}{l}\text { Nondynamic model (one-repeat version) } \\
\text { The palm-side big knuckle of the index of the } \\
\text { flat hand contacts the upper ipsilateral chest, } \\
\text { then the high upper center chest, then the upper } \\
\text { contralateral chest, then the mid contralateral } \\
\text { chest, then the low center chest, then the mid } \\
\text { ipsilateral chest, then the upper ipsilateral chest, } \\
\text { then the high upper center chest, then the upper } \\
\text { contralateral chest, then the mid contralateral } \\
\text { chest, then the low center chest, then the mid } \\
\text { ipsilateral chest. }\end{array}$ & $\begin{array}{l}\text { on it. } \\
\text { ontacts the chest and }\end{array}$ \\
$\begin{array}{l}\text { Inferences: Because there are six locations } \\
\text { arranged equidistantly on the chest, the pathshape } \\
\text { is circular by phonological constraint 5) above. }\end{array}$ & \\
\hline
\end{tabular}




\section{Possible pathshapes: evidence from SignTyp}

A possible objection to pathshape features is that there are too many and too complex pathshapes to be suitable as feature values. Suppose for example, that minimal pairs of signs existed that are distinguished solely by pathshapes which included such values as a random shape with five, six or seven protrusions as shown in Figure 9. There are surely cognitive limits on the shapes that a human being can visually grasp as a unitary phenomenon and distinguish from similar shapes, so if such minimal pairs existed, they would be a strong argument against dynamic pathshape features. The literature on visual perception suggest that complex shapes are decomposed into more elementary shapes using, for example, concave creases as points to cut up a complex shape into smaller less complex shapes (see, e.g., Hoffman 1998, chapter 4). It therefore seems reasonable to expect that the shapes that can function as features would be elementary shapes, or shapes with minimal complexity.

A reasonable expectation would be that except for iconic shapes, possible pathshape features would be roughly congruent with the set of shapes that are named in non-specialized spoken languages. This congruence would be expected because shapes that are named in the vernacular vocabulary of any language should roughly represent the limits of the average person's ability to discriminate between different shapes. For example, English has shape words such as circle, oval, triangle, square, rectangle, line, point, and puddle, but it does not have single word descriptions to distinguish between the paths in Figure 9.
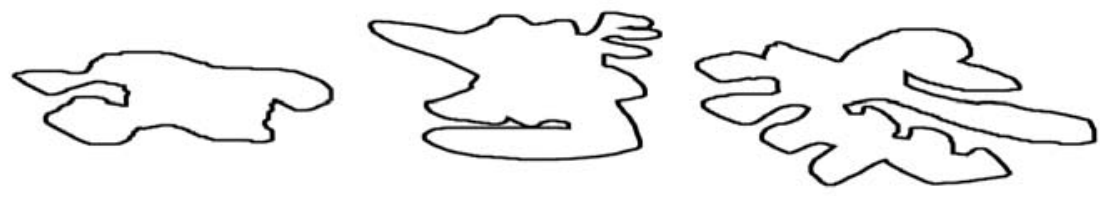

Figure 9. Random complex pathshapes

Of course, complex pathshapes do regularly occur in sign languages, such as pathshapes describing the path of a vehicle or the shape of a complex object. However, they only occur where there is strong iconic support for such a path, usually in classifier predicates. This exceptional behavior can be considered to be because in these signs, the pathshape itself is not stored as a feature. See van der Hulst \& van der Kooij 2006 for further discussion of how iconicity is related to the phonology.

Table 10 and Table 11 shows the specific pathshapes found in SignTyp. The following notes about the data should help in interpreting the tables. 
1. Only two datasets are included: the Long ASL data and the SignPhon NGT data, because these are the only ones that include information on compound status and pathshape.

2. Only non-compound signs were included.

3. Straight then straight and similar locutions in Table 11 could be either the result of drawing successive straight paths in some shape such as a triangle or square or a repeated straight line with the same spatial coordinates. These two types of repetition could not be separated out in the current version of SignTyp.

Table 10. Pathshapes found in SignTyp

\begin{tabular}{lllll}
\hline $\begin{array}{l}\text { Dataset \& } \\
\text { Language }\end{array}$ & $\begin{array}{l}\text { Total } \\
\text { signs }\end{array}$ & $\begin{array}{l}\text { Noncompound } \\
\text { signs }\end{array}$ & $\begin{array}{l}\text { Signs with } \\
\text { pathshapes }\end{array}$ & $\begin{array}{l}\text { \% of signs with } \\
\text { pathshapes }\end{array}$ \\
\hline Long ASL & 1549 & 1120 & 151 & $9.7 \%$ \\
SignPhon NGT $^{14}$ & 3080 & 2811 & 859 & $27.9 \%$ \\
\hline Totals & 4629 & 3931 & 1428 & $15.8 \%$ \\
\hline
\end{tabular}

Table 11. Types of pathshape by dataset

\begin{tabular}{llllll}
\hline Path group & Pathshape & $\begin{array}{l}\text { Long } \\
\text { ASL }\end{array}$ & $\begin{array}{l}\text { SignPhon } \\
\text { NGT }\end{array}$ & Percent & $\begin{array}{l}\text { Running } \\
\text { total } \\
\text { percent }\end{array}$ \\
\hline $\begin{array}{l}\text { arcs \& } \\
\text { circles }\end{array}$ & arc & $8.0 \%$ & $55.8 \%$ & $48.0 \%$ & $48.0 \%$ \\
& circle & $60.8 \%$ & $30.4 \%$ & $35.4 \%$ & $83.4 \%$ \\
& 2 circles & $2.4 \%$ & $0.0 \%$ & $0.4 \%$ & $83.8 \%$ \\
& 3 circles & $0.0 \%$ & $0.2 \%$ & $0.1 \%$ & $83.9 \%$ \\
& semicircle & $13.6 \%$ & $0.0 \%$ & $2.2 \%$ & $86.2 \%$ \\
7 shape & & $0.0 \%$ & $0.2 \%$ & $0.1 \%$ & $86.3 \%$ \\
cross or x & & $6.4 \%$ & $0.2 \%$ & $1.2 \%$ & $87.5 \%$ \\
shape & & & & & \\
Iconic & & $1.6 \%$ & $4.4 \%$ & $4.0 \%$ & $91.4 \%$ \\
L & & $0.8 \%$ & $0.0 \%$ & $0.1 \%$ & $91.6 \%$ \\
Zigzag & & $6.4 \%$ & $0.6 \%$ & $1.6 \%$ & $93.1 \%$
\end{tabular}




\begin{tabular}{|c|c|c|c|c|c|}
\hline \multirow{2}{*}{$\begin{array}{l}\text { Multiple } \\
\text { straight } \\
\text { paths }\end{array}$} & $\begin{array}{l}\text { Straight } \\
\text { then straight }\end{array}$ & $0.0 \%$ & $3.9 \%$ & $3.3 \%$ & $96.4 \%$ \\
\hline & $\begin{array}{l}\text { Straight } \\
\text { then straight } \\
\text { then straight }\end{array}$ & $0.0 \%$ & $0.3 \%$ & $0.3 \%$ & $96.7 \%$ \\
\hline \multirow{7}{*}{$\begin{array}{l}\text { circular } \\
\text { \& straight } \\
\text { paths }\end{array}$} & $\begin{array}{l}\text { arc then } \\
\text { straight }\end{array}$ & $0.0 \%$ & $2.2 \%$ & $1.8 \%$ & $98.6 \%$ \\
\hline & $\begin{array}{l}\text { straight then } \\
\text { arc }\end{array}$ & $0.0 \%$ & $0.9 \%$ & $0.8 \%$ & $99.3 \%$ \\
\hline & $\begin{array}{l}\text { straight then } \\
\text { arc then } \\
\text { straight }\end{array}$ & $0.0 \%$ & $0.2 \%$ & $0.1 \%$ & $99.5 \%$ \\
\hline & $\begin{array}{l}\text { circle then } \\
\text { straight then } \\
\text { straight }\end{array}$ & $0.0 \%$ & $0.2 \%$ & $0.1 \%$ & $99.6 \%$ \\
\hline & $\begin{array}{l}\text { straight then } \\
\text { circle }\end{array}$ & $0.0 \%$ & $0.2 \%$ & $0.1 \%$ & $99.7 \%$ \\
\hline & $\begin{array}{l}\text { arc then } \\
\text { straight then } \\
\text { straight }\end{array}$ & $0.0 \%$ & $0.2 \%$ & $0.1 \%$ & $99.9 \%$ \\
\hline & $\begin{array}{l}\text { straight then } \\
\text { arc then arc }\end{array}$ & $0.0 \%$ & $0.2 \%$ & $0.1 \%$ & $100.0 \%$ \\
\hline
\end{tabular}

Examples of iconic paths include: outline of a statue, oblong shape, contours of a Christmas tree, shape of mountain, outline of priest's clothing, shape of figure, question mark, and rectangle omitting one side.

Excluding the iconic pathshapes, the inventory of paths is relatively small and easily distinguished. It is not necessary to characterize the difference between two different circular/oval paths or variations in the productions of a cross shape; and the exact number of turns in a zigzag line is not counted. After all, the pathshape features, as phonological entities, generalize over an infinite number of variants; their denotations are not a precise iconic description of any specific actual path.

Of the 25 signs in SignPhon with more complex paths, most could probably be explained as having iconic elements, but further information is needed to determine this. The variation between datasets in both number 
and type of pathshapes is also left unexplained here, but this would certainly be an important area for future research, to determine if these variations are true crosslinguistic variations, or effects of differences in coding strategies. Overall however the data supports the argument that pathshape features in signs are relatively simple and have a manageable number of values. There is therefore good reason to believe that possible pathshapes in signs form a small closed set which can function as features.

\section{Conclusion}

To summarize, the first part of this chapter showed that dynamic features, in conjunction with the omission of characteristics that are not phonological, make representations extremely simple. The second part showed that dynamic features for pathshape must be a part of the phonological model because without pathshape features, representations become psychologically unrealistic. A nondynamic model has the following problems:

1. Too many features, too much detail. A nondynamic model must use many more features, specified in much greater detail than in the dynamic model. The kind of detail used requires high levels of discrimination between similar features: for example the signer must discriminate between high upper center chest and mid ipsilateral chest.

2. Arbitrary features. There is nothing in the physical sign which indicates that the index finger has a special relationship with the chest location compared to the other digits or the palm or that the six selected locations are special.

3. Indeterminate number of representations for one sign. The nondynamic model has to repeat the six locations exactly as often as they are repeated, since it cannot use a dynamic [repeat] feature, so different numbers of repetitions generate different representations.

4. Segment strings are too long. Representations without [repeat] result in inordinately long location strings and other features.

5. Magical inferences. The nondynamic model requires inferences which are not phonetically sensible (if there are three locations, the path is angular, if there are six, the path is circular).

6. Structural problems. Features which do not change when the hand moves to a new location must either be repeated for each segment or moved to a suprasegmental position and some sort of spreading constraint must be invoked. 
Thus, even though it is mathematically simpler to specify a path in terms of a finite number of points, it is much more likely that we mentally perceive and record the path as one of a small set of shapes: an arc, circle, spiral, cross, 7, or zigzag, with a default unspecified value of straight line for the majority of signs.

An additional result of accepting dynamic features is that a single segment representation becomes almost mandatory ${ }^{15}$. This is because, as Padden \& Perlmutter (1987) pointed out, it makes no sense to attach a dynamic feature to a particular segment when it applies to the entire sign. For example, if a circling pathshape occurs over the entire sign, but the representation had multiple segments, to which segment should the circling feature be attached? The presence of dynamic features therefore means that a single segment model is highly favored. Such a model might be similar to van der Hulst's (1993) temporally sequenced single segment. Or it could be like Channon's (1996, 2002a) segment which does not have internal temporal sequencing elements but assumes that whatever sequencing occurs is a result of dynamic features. Other arguments must be found to decide between these two models, and are not addressed here.

Hayes' suggestion, then, to eliminate dynamic features cannot be fully followed, since at least pathshape and repetition are required. This opens the door to admitting additional dynamic characteristics. One might even say that the presence of two dynamic features raises an expectation that others also occur, since it would be surprising if only two features of an entire possible type were used. In particular, direction features and handshape opening/ closing features become more likely and more reasonable.

If pathshapes had to be the result of interpolations between locations, it would mean that the mind is functioning like a digital machine in all respects- painstakingly linking locations together by calculating the paths between them. As an analogy, one might compare the nondynamic model to the connect-the-dots drawings given to children. A figure (e.g. of some kind of animal) is represented in terms of numbered dots and the child then has to connect the numbers in the correct order to see the figure. A nondynamic model claims that we mentally represent shapes in terms of a set of numbered points, rather than directly in terms of the shape itself. But if, as argued here, the mind is using pathshapes as basic concepts (as 'gestalt'), it suggests that at least elementary, basic shapes can and should be represented holistically. 


\section{Notes}

1. This material is based upon work supported by the National Science Foundation under Grant No. 0544944 to both authors. Any opinions, findings, conclusions or recommendations expressed in this material are those of the author and do not necessarily reflect the views of the National Science Foundation.

2. Pathshape could be considered a multivalent feature with values such as [arc/zigzag/circle] or a class node with dependent features [arc], [zigzag] and [circle]. Here, pathshape is referred to as a class node, but nothing in this chapter hangs on this distinction. Note that referring to a feature using a word such as zigzag does not mean that that shape features are mentally represented in terms of 'words'. Rather the assumption is that the mental representation is in terms of articulatory instructions, and/or in terms of a 'perceptual image' of the shape. Of course, the same applies to spoken language features for which we also use 'words': here too the features are articulatory instructions and/or acoustic images.]

3. Of course, we can also visualize ourselves walking down the path over time in a particular direction as though watching a movie, but this is not what is meant here, but rather the direct immediate map-like perception of a path as a whole with a certain shape and direction. The difference between these two means of perception is the difference between driving by following instructions one at a time (turn left, then turn right.....) and driving by having a picture in one's mind of the entire path to be followed as well as its relationship with the surrounding areas.

4. An interesting question for future research is whether the acquisition of dynamic features might be slightly delayed relative to static features. If the analogy in footnote 3 is appropriate, it often takes time to build up a map of an area, and many drivers begin by learning a route as a series of turns. Thus it might also be true that children might learn their first few sign as a series of arbitrary points in space. As they acquire the concepts of path and direction, these might then be used to acquire further signs, as well as to reanalyze those already acquired.

5. Characteristic: any feature can be a characteristic, but not all characteristics are features. Used when there is some doubt as to whether or not something is actually a phonological feature, or when considering both phonetic and phonological elements.

6. An extended handshape has no knuckles bent, a bent handshape has only the large knuckle bent, a hooked handshape has only the two smaller knuckles bent, a curved handshape has all knuckles flexed but not completely closed, and a closed handshape has all knuckles fully bent or closed. 
7. For example, MOTHER contacts the chin with the thumb. Although some handshapes are ruled out, the fist and open hand seem almost equally likely candidates, so it is probably necessary to list something about the handshape. In this case, it may be sufficient to list spreading, because the fist cannot spread. Another example would be the two different forms of YESTERDAY, one with the fist, and the other (initialized) with a fist with the pinky extended. For this sign it would be necessary to exclude the open hand and for the initialized form to list the extension of the pinky.

8. The sign may also be slightly off-centered to the contralateral side for efficiency - to keep the elbow closer to the body. If this is correct, a simple phonetic constraint could produce this off-centered location.

9. HAPPY: The palmside of the hand with all digits extended and unspread repeatedly brushes upward on the chest with initial contact on the chest followed by an upward and outward movement which moves the hand out into space.

10. It should now be clear to the reader why other pathshapes are not considered - it would add more pathshapes to distinguish with different numbers of locations without changing the argument.

11. Notice that an additional arbitrary factor here is that these locations depend in part on the size of the particular hand and chest. For example, someone with an especially large chest and small hand might move the hand only in the mid area of the chest and therefore require a different set of features than the ones specified in the example.

12. If the model does not allow the feature omissions discussed in 3.1.2, then features for orientation, handshape, and other values would have to be added as well, further increasing the number of nodes.

13. PANCAKE (also KITCHEN): back of the strong hand contacts palm of weak hand, then turns over so that the palms contact. The handshape is all digits extended and unspread.

14. SignPhon was the only dataset that also listed the default straight path. This occurred in 1766 (62.8\%) of all non-compound signs. An additional 186 signs $(6.6 \%)$ of all non-compound signs had no path. These would be signs with hand internal motion, orientation change or similar situations where the action of the sign does not involve moving the hand from one location to another. This information is not available for the Long dataset which did not distinguish between straight paths and no path.

15. Almost mandatory: it might be possible to get around this by using a hybrid model with some kind of suprasegmental root node and internal segments. For example, in a manner similar to Sandler's (1993) spreading handshape and location features, it could be argued that circling spreads to all segments. 


\section{References}

Brentari, Diane.

1998 A prosodic model of sign language. Cambridge MA: MIT. Channon Crain, Rachel.

1996 Representing a sign as a single segment in American Sign Language. In Green and Motopanyane (ed.), Proceedings of the 13th Eastern States Conference on Linguistics, 46-57. Cornell University, Ithaca NY.

Channon, Rachel.

2002a Signs are single segments: Phonological representations and temporal sequencing in ASL and other sign languages. University of Maryland at College Park Dissertation. http://www.ling.umd. edu/publications/dissertations/

2002b "Beads on a String? Representations of repetition in spoken and signed languages.” In R. Meier, K. Cormier, \& D. Quinto-Pozos, (eds.), Modality and Structure in Signed and Spoken Languages. Cambridge, UK: Cambridge University, pp. 110-148.

Corina, David P.

1993 To branch or not to branch: Underspecification in American Sign Language handshape contours. In Coulter, 63-95.

Crasborn, Onno.

2001 Phonetic Implementation of Phonological Categories in Sign Language of the Netherlands. Doctoral dissertation, Leiden University, The Netherlands.

Brentari, Diane \& Petra Eccarius.

2011 When does a system become phonological? Potential sources of handshape contrast in sign languages. In R. Channon \& H. van der Hulst (eds.). Formational Units in Sign Languages. Sign Language Typology Series Vol. 3. Nijmegen / Berlin: Ishara Press / Mouton de Gruyter.

Hayes, B.

1993 Against movement: comments on Liddell's article. In G. Coulter (ed.) Phonetics and phonology, vol. 3: current issues in ASL phonology. San Diego CA: Academic Press. Pp. 213-226

Hansen.

2011 ASL Movement Phonemes and Allophones. In R. Channon \& H. van der Hulst (eds.). Formational Units in Sign Languages. Sign Language Typology Series Vol. 3. Nijmegen / Berlin: Ishara Press / Mouton de Gruyter. 
Hoffman, Donald.

1998 Visual Intelligence. How We Create What We See. New York/ London: W.W. Norton \& Company.

Liddell, Scott.

1984 THINK and BELIEVE: Sequentiality in American Sign Language Signs, Language 60. 2, 372-399.

Liddell, Scott and Johnson, R. E.

1989 American Sign Language: The phonological base. Sign Language Studies, 64, 195-277.

Mak, Joe \& Gladys Tang

2011 Movement types, repetition and feature organization in Hong Kong Sign Language. In R. Channon \& H. van der Hulst (eds.). Formational Units in Sign Languages. Sign Language Typology Series Vol. 3. Nijmegen / Berlin: Ishara Press / Mouton de Gruyter.

Padden, Carol A. and David M. Perlmutter

1987 American Sign Language and the Architecture of Phonological Theory. Natural Language and Linguistic Theory, 5, 3, Aug, 335375.

Sandler, W.

1993 Linearization of phonological tiers in ASL. In G. Coulter (ed.) Current issues in ASL phonology. New York: Academic Press, 103-29.

van der Hulst, Harry

1993 Units in the Analysis of Signs. Phonology 10, 209-41.

van der Hulst, Harry and Els van der Kooij

2006 Phonetic implementation and phonetic pre-specification in sign language phonology. In Goldstein, Whalen \& Best, Laboratory Phonology 8. New York: De Gruyter.

Van der Kooij, Els.

2002 Phonological Categories in Sign Language of the Netherlands: The Role of Phonetic Implementation and Iconicity. Doctoral dissertation, Leiden University, The Netherlands. 